\title{
Long-wavelength III-V/silicon photonic integrated circuits
}

\author{
G. Roelkens, B. Kuyken, F. Leo, N. Hattasan, E. \\ Ryckeboer, M. Muneeb, C. Hu, A. Malik, Z. Hens, R. Baets \\ Photonics Research Group - NB-Photonics \\ Ghent University-IMEC, \\ B-9000 Gent, Belgium
}

\author{
Y. Shimura, F. Gencarelli, B. Vincent, R. Loo, P. Verheyen, \\ G. Lepage, J. Van Campenhout \\ imec, Kapeldreef 75, B-3001 Leuven, Belgium \\ L. Cerutti, J.B. Rodriguez, E. Tournié \\ NanoMIR group, University of Montpellier 2, France
}

\author{
X. Chen, M. Nedeljkovic, G. Z. Mashanovich \\ Optoelectronics Research Centre, Faculty of Physical and \\ Applied Sciences, University of Southampton, \\ Southampton, SO17 1BJ United Kingdom \\ X. Liu, R. Osgood \\ Department of Electrical Engineering. Columbia Univeristy, \\ New York 10027, USA \\ W.M.J. Green \\ IBM T.J. Watson Research Center, 1101 Kitchawan Road, \\ Yorktown Heights, New York 10598, USA
}

\begin{abstract}
We review our work in the field of short-wave infrared and mid-infrared photonic integrated circuits for applications in spectroscopic sensing systems. Passive silicon waveguide circuits, GeSn photodetectors, the integration of III-V and IV-VI semiconductors on these circuits, and silicon nonlinear optics are discussed.
\end{abstract}

Keywords: photonic integration; spectroscopic sensing; silicon photonics

\section{INTRODUCTION}

Many gases that we need to detect or monitor in our environment have bands of absorption lines in the short-waveinfrared (SWIR - 1.4-3 $\mu \mathrm{m}$ ) and mid-wave-infrared (MWIR 3$8 \mu \mathrm{m})$ [1]. These absorption lines form a "fingerprint" for a particular molecule; hence SWIR/MWIR spectroscopic sensing systems allow detecting the presence (and concentration) of specific molecules. Currently available systems are either table-top-size instruments or composed out of discrete opto-electronic components. Therefore, these systems are expensive, not handheld, and power hungry. Photonic integration, now a well developed technology for the realization of complex photonic integrated circuits for communication applications, could alleviate the issues that current spectroscopic sensing systems face. Combined with high-volume manufacturing capabilities, such integrated systems might be ubiquitously deployed in industrial process control, monitoring our environment and probing our exhaled breath for disease markers. Photonic integration on a silicon platform can leverage the huge investments made in CMOS fabrication technology in order to realize photonic integrated circuits for SWIR/MWIR applications. In this paper we review our recent work in this field. This includes the realization of passive SWIR/MWIR silicon photonic integrated circuits, the realization of GeSn-based photodetectors on a silicon substrate, the heterogeneous integration of GaSb-based optoelectronic components on this platform and the use of silicon nonlinear optics for the generation and detection of midinfrared radiation.

\section{PASSIVE FUNCTIONALITY AND GESN PHOTODETECTORS}

Low-loss silicon waveguides are of key importance for the realization of integrated spectroscopic sensing systems. The silicon-on-insulator waveguide platform is transparent up to approximately $4 \mu \mathrm{m}$, above which the absorption of the $\mathrm{SiO}_{2}$ buried oxide layer becomes prohibitive. Therefore it provides an interesting platform to develop spectroscopic sensing systems on. In parallel, literature is emerging, which addresses silicon-based waveguide platforms for above $4 \mu \mathrm{m}$ operation, including free-standing silicon [2], silicon-on-sapphire [3] and germanium-on-silicon [4]. The waveguide structures used in this paper are based on either a $220 \mathrm{~nm}$ or $400 \mathrm{~nm}$ thick silicon device layer on a $2 \mu \mathrm{m}$ buried oxide layer, and the devices are fabricated in multi-project wafer runs, alongside silicon photonic integrated circuits for near-infrared applications. Waveguide losses down to $0.5 \mathrm{~dB} / \mathrm{cm}$ in the $2-2.5 \mu \mathrm{m}$ wavelength range are obtained for single-mode $220 \mathrm{~nm}$-thick silicon strip waveguides [5]. For 400nm-thick silicon waveguides $3 \mathrm{~dB} / \mathrm{cm}$ waveguide losses are obtained at $3.8 \mu \mathrm{m}$ wavelength, a fraction of which is due to substrate leakage due to the $2 \mu \mathrm{m}$ buried oxide layer thickness [6]. On the same platform fiber-to-chip diffractive grating couplers were developed that reach $-3.8 \mathrm{~dB}$ coupling efficiency in the shortwave infrared ( $3 \mathrm{~dB}$ bandwidth $80 \mathrm{~nm}$ ) [5]. On this platform complex integrated spectrometers in the form of arrayed waveguide gratings and planar concave gratings, both for the

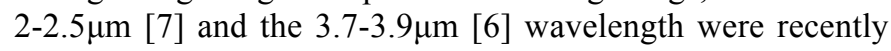
demonstrated. Remarkably, these components are close to matching the performance of well-developed near-infrared wavelength (de)multiplexers that have been optimized via many device fabrication-development cycles. 


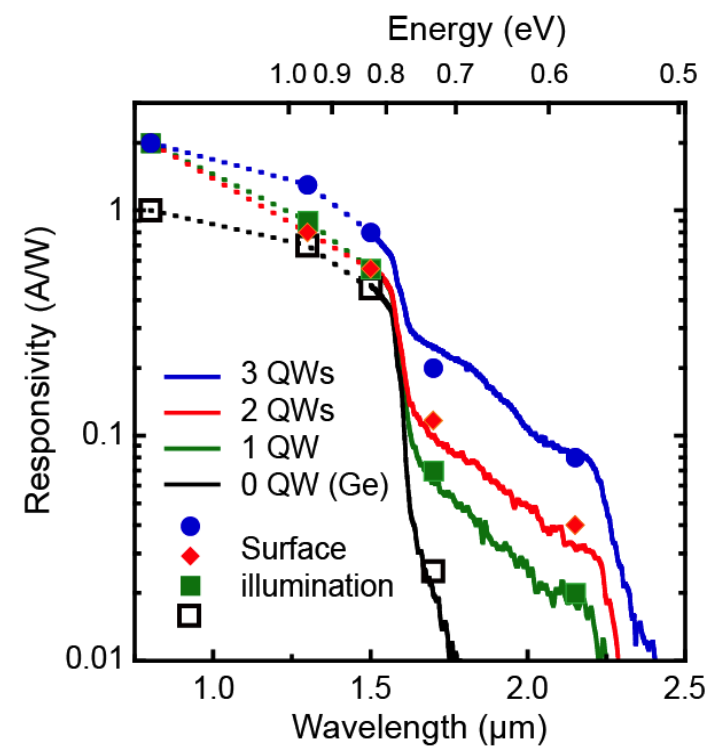

Figure 1. Responsivity of multi-quantum well $\mathrm{Ge}_{0.9} \mathrm{Sn}_{0.1} / \mathrm{Ge}$ photoconductors on silicon substrate

In order to realize complete integrated spectrometers, there is a need for integrated long-wavelength photodetectors. While III-V semiconductor (GaSb-based and InP-based) and IV-VI / II-VI semiconductors ( $\mathrm{PbS}, \mathrm{PbSe}, \mathrm{HgCdTe}$ ) are nowadays used as stand-alone long-wavelength detectors, Ge-based materials can also be envisioned for this function. Indeed, by adding Sn to the Ge-matrix, the bandgap of the Ge is reduced, unlocking the possibility of monolithically integrated photodetectors in the short-wave infrared. Recently we demonstrated $\mathrm{Ge}_{0.9} \mathrm{Sn}_{0.1}$ multi-quantum well photoconductors integrated on a silicon substrate with a $2.4 \mu \mathrm{m}$ wavelength cutoff wavelength [8], as shown in Figure 1. In order to go far beyond this cut-off wavelength, either new developments are needed in terms of the amount of Sn that can be incorporated in the Ge-matrix or III-V semiconductors and IV-VI semiconductors need to be heterogeneously integrated on the silicon platform.

\section{HETEROGENEOUS INTEGRATION}

Heterogeneous integration is currently being considered by several research groups and industrial players as an enabling technology for integrating a near-infrared laser source on the silicon photonics platform [9]. Molecular bonding, adhesive bonding and metallic bonding are being considered. Similar technologies can also be used for bringing III-V semiconductors with an operating wavelength in the midinfrared onto silicon photonics. We have demonstrated the heterogeneous integration GaSb-based photodetector arrays (cut-off wavelength $2.5 \mu \mathrm{m}$ ) on silicon spectrometers by means of DVS-BCB adhesive bonding, as shown in Figure 2 [7]. Also, the integration of arrays of single wavelength lasers on silicon is under study, which has yielded the first SWIR $\mathrm{GaSb}$ lasers integrated on and coupled to silicon waveguides [10]. In addition to the layer transfer of III-V semiconductor epi-layers, also the integration of IV-VI materials onto silicon waveguide circuits is also being studied. These materials take the form of colloidal nanoparticles that are synthesized in solution and coated on silicon waveguides to allow the realization of cheap and sensitive infrared photodetectors. Currently we are focusing on the realization of $\mathrm{PbS}$ and $\mathrm{HgTe}$ colloidal nanocrystal photoconductors with demonstrated cutoff wavelengths of $2.4 \mu \mathrm{m}$ and $2.8 \mu \mathrm{m}$ respectively and responsivities in the range of several $100 \mathrm{~A} / \mathrm{W}$ due to the high internal gain of these devices [11].

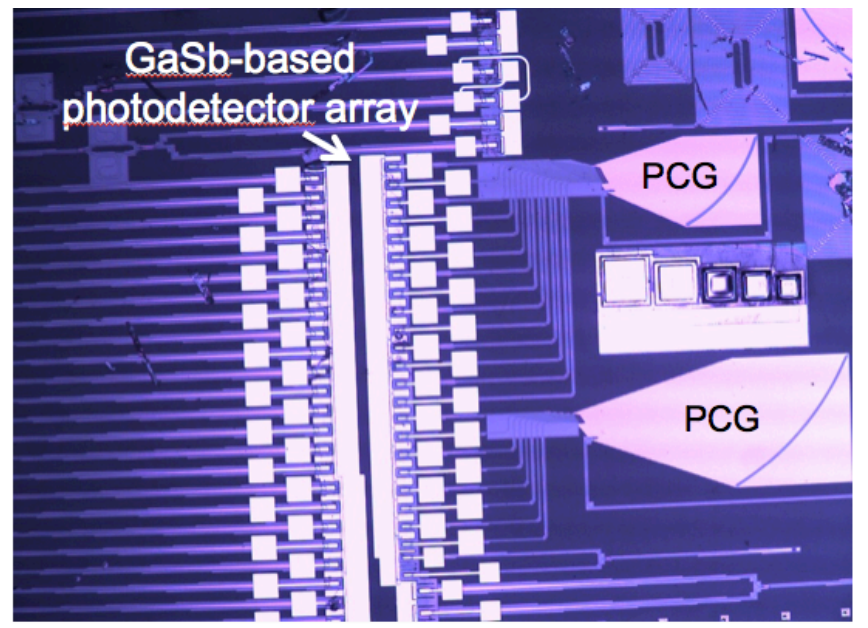

Figure 2. Integrated short-wave infrared spectrometer comprising a silicon spectrometer and heterogeneously integrated GaSb-based photodetector array

\section{SWIR/MWIR SILICON NONLINEAR OPTICS}

Another attractive proposition for silicon-based integrated spectroscopic optical functions is the use of the strong $\chi^{(3)}$ nonlinearity of silicon, combined with the high refractive index contrast available on this platform. The latter allows for a tight confinement of the optical field and allows for broad dispersion engineering of these waveguides, two critical features that are required for efficient nonlinear interactions. We focus on the use of four-wave mixing processes either to generate SWIR/MWIR radiation or to up-convert this midinfrared radiation to the near-infrared, where they can be detected by well-developed uncooled photodetectors. While nonlinear optics on a silicon platform at telecommunication wavelengths is not efficient, it is, however so in the shortwave infrared. This is related to the absence of parasitic twophoton absorption above pump wavelengths of $2.2 \mu \mathrm{m}$, making the silicon platform nearly ideal for nonlinear optics, featuring nonlinear parameters that are orders of magnitude higher than those obtained in optical fibers. Recently we have demonstrated wavelength conversion over 1.2 octaves from a telecom-wavelength signal to a $3.6 \mu \mathrm{m}$ idler signal using a $2.19 \mu \mathrm{m}$ pump [12]. A conversion gain of about $10 \mathrm{~dB}$ was estimated for this experiment. This result shows that fourwave mixing is a viable mechanism of generating tunable midinfrared radiation, either by using a tunable telecomwavelength signal or by tuning the pump wavelength. Also, the upconversion of mid-infrared radiation into the telecom wavelength range was demonstrated as shown in Figure 3 [13]. Next to wavelength conversion, supercontinuum generation from $1.6-2.55 \mu \mathrm{m}$ [14] and optical parametric 
oscillators in the SWIR based on $>50 \mathrm{~dB}$ parametric gain in a $2 \mathrm{~cm}$ long silicon waveguide spiral was demonstrated on the same platform [15].

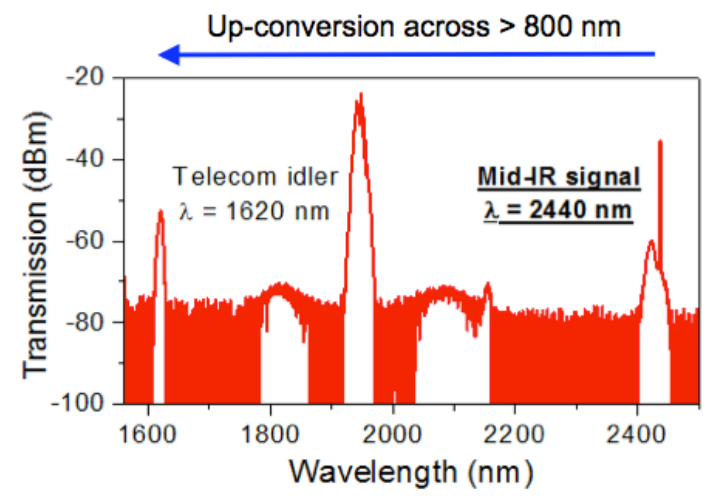

Figure 3. Wavelength conversion through four-wave mixing. Dispersion engineering of the silicon waveguide allows spectral translation to and from the telecom wavelength range

\section{CONCLUSIONS}

Long-wavelength silicon photonic integration is an emerging research field that leverages the broad technology base that was developed for near-infrared photonic integrated circuits. It allows the realization of integrated spectroscopic systems that can be ubiquitously deployed due to their low-cost and compact and rugged design.. In the last year several device demonstrations have been realized showing the potential of this technology. Outside of the spectroscopy domain, other application areas can be identified such as optical communication in the $2 \mu \mathrm{m}$ wavelength range [16], free-space optical communication and infrared (hyper-spectral) imaging systems.

\section{REFERENCES}

[1] Crowder et al.,'Infrared methods for gas detection", in Mid-infrared semiconductor optoelectronics, Springer (2006)

[2] Y. Xia et al., "Suspended silicon ring resonator for mid-IR application," Optics Letters 2013

[3] R. Shankar et al., "Integrated high-quality factor silicon-on-sapphire ring resonators for the mid-infrared," Applied Physics Letters 102, 051108 (2013)

[4] Y. Chang et al., "Low-loss germanium strip waveguides on silicon for the mid-infrared," Optics Letters 37(14), p. 2883-2885 (2012)

[5] N. Hattasan, B. Kuyken, F. Leo, E.M.P. Ryckeboer, D. Vermeulen, G. Roelkens, High-efficiency SOI fiber-to-chip grating couplers and lowloss waveguides for the short-wave infrared,IEEE Photonics Technology Letters, 24(17), p.1536-1538 (2012)

[6] M. Muneeb et al., "Demonstration of silicon-on-insulator mid-infrared spectrometers operating at 3.8um," Optics Express 2013

[7] E.M.P. Ryckeboer, A. Gassenq, M. Muneeb, N. Hattasan, S. Pathak, L. Cerutti, J.-B. Rodriguez, E. Tournie, W. Bogaerts, R. Baets, G. Roelkens, Silicon-on-insulator spectrometers with integrated GaInAsSb photodiodes for wide-band spectroscopy from 1510 to $2300 \mathrm{~nm}$, Optics Express, 21(5), p. 6101-6108 (2013)

[8] A. Gassenq, F. Gencarelli, J. Van Campenhout, Y. Shimura, R. Loo, G. Narcy, B. Vincent, G. Roelkens, GeSn/Ge heterostructure short-wave infrared photodetectors on silicon, Optics Express, 20(25), p.2729727303 (2012)

[9] G. Roelkens, L. Liu, et al.,'III-V/silicon photonics for on-chip and interchip optical interconnect," Laser Photonics Rev. P.DOI: 10.1002/lpor.200900033 (2010).

[10] N. Hattasan, A. Gassenq, L. Cerutti, J.B. Rodriguez, E. Tournie, G. Roelkens, The study of integrated thin film GaSb-based Fabry Perot lasers: towards a fully integrated spectrometer on a Silicon-On-Insulator waveguide circuit, SPIE Photonics West 2013

[11] C. Hu, A. Gassenq, Yolanda Justo, Sergii Yakunin, Wolfgang Heiss, Zeger Hens, G. Roelkens, Short-wave Infrared Colloidal Quantum Dot Photodetectors on Silicon, SPIE Photonics West 2013

[12] B. Kuyken, P. Verheyen, P. Tannouri, J. Van Campenhout, R. Baets, G. Roelkens, W. M.J. Green, Mid-Infrared Generation by Frequency Down-Conversion Across 1.2 Octaves in a Normally-Dispersive Silicon Wire, submitted to CLEO 2013

[13] X. Liu, B. Kuyken, G. Roelkens, R. Baets, R. M. Osgood Jr., W. M. J. Green, "Bridging the Mid-Infrared-to-Telecom Gap with Silicon Nanophotonic Spectral Translation", Nature Photonics, p.667671 (2012).

[14] B. Kuyken, X. Liu, R. M. Osgood Jr., R. Baets, G. Roelkens, W. M.J. Green, Mid-infrared to telecom-band supercontinuum generation in highly nonlinear silicon-oninsulator wire waveguides,Optics Express, 19(21), p.20172-20181 (2011)

[15] B. Kuyken, X. Liu, R. M. Osgood Jr., R. Baets, G. Roelkens, W. M. J. Green, A Silicon-Based Widely Tunable Short-Wave Infrared Optical Parametric Oscillator, Optics Express 21(5), p.5931-5940 (2013)

[16] N. MacSuibhne, Z. Li, B. Baeuerle, J. Zhao, J. Wooler, S. Alam, F. Poletti, M. Petrovich, A. Heift, I. Giles, B. Palsdottir, L. Gruner-Nielsen, R. Phelan, J. O'Caroll, B. Kelly, D. Murphy, A. Ellis, D. Richardson, F. Garcia Gunning, "Wavelength division multiplexing at 2um," PDP Th3A3, ECOC 2012 